\title{
A Data Technology Oriented to Information Fusion to Build an Intelligent Accounting Computerized Model
}

\author{
Ziyan Xu (iD and Wenxue Zhou \\ Tangshan Normal University, Tangshan 063000, China \\ Correspondence should be addressed to Ziyan Xu; xzy110015@tstc.edu.cn
}

Received 19 October 2021; Revised 10 November 2021; Accepted 18 November 2021; Published 8 December 2021

Academic Editor: Le Sun

Copyright (c) 2021 Ziyan Xu and Wenxue Zhou. This is an open access article distributed under the Creative Commons Attribution License, which permits unrestricted use, distribution, and reproduction in any medium, provided the original work is properly cited.

\begin{abstract}
The development of management accounting promotes the integration of business and finance, and with the continuous development of information technology and the advent of the era of big data, the development of corporate financial informatization provides tools for the integration of business and finance. This paper improves the big data technology, improves the traditional accounting process, combines the big data technology to build a computerized accounting system, and obtains scientific and effective accounting information processing results through intelligent big data processing. The design goal of the enterprise accounting management system is that the system can efficiently complete the enterprise cost budget accounting work after the design is completed and ensure the normal and stable progress of the enterprise cost budget accounting work. Through the experimental research results, it can be known that the computerized intelligent accounting system based on big data technology constructed in this article has certain effects.
\end{abstract}

\section{Introduction}

In contemporary society, various system reconstruction methods are emerging one after another, but they have never really solved this problem. Since the advent of the REA model, the construction of an accounting information system based on REA can provide the company's stakeholders and auditors with real-time, accurate, multilevel, personalized management decision information, audit information, etc. Moreover, it satisfies the information needs of more people, thereby transforming the accounting information system from the traditional focus on financial status, operating results, and cash flow to real-time control and feedback of the enterprise management process. Furthermore, it enables the system to develop into an intelligent accounting information system that can provide information based on user choices to support management decisions and so forth [1].

With the development of network computer technology, the user-friendly operation interface of the commonly used Windows system has accelerated the use of accounting software in the financial management department of enterprises and has slowly extended it to other departments. This good application situation also makes the academic and practical circles begin to consider the issue of information integration between different departments to avoid duplication of work between departments and improve work efficiency. For example, the accounting department is linked with the cost accounting of the production department and the sales management of the sales department to realize the integration of departmental subsystems, and the accounting information system gradually rises from this [2]. However, the accounting information system at this time belongs to the accounting system dedicated to the financial department and cannot provide management decision information, which is more restrictive at the technical and conceptual levels. The processing of data in the system application only serves as a postevent record and feedback function, and it is difficult for it to play the role of pre-prediction and in-event control [3].

Since then, with the vigorous development of databases, the flexibility of information systems has improved, and data 
security, analysis, and processing capabilities have made great progress. Enterprise-level management information systems represented by ERP have developed rapidly, and their essence is to break departmental barriers; expand the scope of the information system; connect the upstream and downstream of the supply chain; realize the integration and coordination of the enterprise's finance, personnel, sales, supply, production, etc.; and effectively control the entire process of enterprise operation. In the system of integrated information management, the accounting supervision function of the enterprise is only one of the submodules. With the exquisite network technology, the global IT field has led to a wave of industrial reforms; the development of e-commerce has also swept the traditional accounting business model field, causing companies to rethink the shortcomings of their traditional system architecture and processes; and a series of new system reconstruction method is gradually applied to the accounting information system. The system reconstruction method is gradually applied to the accounting information system. It is widely known as the accounting information system based on the REA model to be introduced in this article, which can realize real-time processing of monitoring information in a network environment and multilevel output information to meet different requirements of users.

Based on the above analysis, this article combines big data technology to construct a computerized accounting system obtains scientific and effective accounting information processing results through intelligent big data processing, and improves accounting management efficiency on this basis.

\section{Related Work}

The implementation path of business-finance integration relies on the integration of various management accounting tools. The literature [4] believes that management accounting information systems should be based on solid corporate management data to enable comprehensive budget management, centralized capital management, cost control, performance evaluation, and other business modules to operate and develop efficiently and smoothly, support the application of management accounting, fully realize the organic integration of accounting and business, and ultimately achieve the goal of value creation. The literature [5] believes that, in the era of big data, traditional management accounting is mainly based on small data calculations and that management accounting tools are separated from each other and lack business-financial integration ideas. Therefore, it believes that it is necessary to integrate modern information technology, management accounting thoughts, and financial accounting theories to form an integrated structure of financial services. In summary, the advanced integrated concept of information system construction has enabled the implementation of management accounting tools such as balanced scorecards, comprehensive budget management, and other management tools. Secondly, the construction process of the management accounting information system is the process of reorganizing and optimizing the business and financial processes. The literature
[6] believes that the success of process management depends on whether financial management and business process redesign are integrated. The literature [7] finds that the lack of support from the management accounting system is the main reason for the failure of business process management. In the practice of process management, the management accounting system can solve the problem of information efficiency. In the process of the organization's operation chain management or supply chain management, for the improvement of organizational performance, appropriate information technology methods can be used to supplement the management accounting system, and the organizational structure, responsibilities, and permissions can be redivided, which will produce better output benefits. Literature [8] believes that management accounting research needs to take informationization as the basis and management integration as a prerequisite. At the same time, its needs to involve the integration of management accounting and business control, financial accounting reports, human resource management, and other systems, as well as the integration of various application models and tools of management accounting itself. In this way, the theoretical system of management accounting can be enriched, and the guidance of management accounting theory to practice can be enhanced at the same time. Finally, the construction of information systems can achieve the effect of business-finance integration on business control. The literature [9] believes that when an enterprise establishes an information system, business control should be embedded in the information system at the same time. The effective operation of the information system control can ensure the effectiveness of the enterprise's internal control.

Literature [10] believes that a management information system is an information system that connects various internal organizations. The internal operation process of the system should be that the software supplier extracts the complete business process from the mature and perfect enterprise to evolve, and allows other users to reorganize the relevant process through standardized learning. Literature [11] believes that, by using information technology correctly under the key role of industry-finance integration, modern enterprises can remain invincible in the external social environment and obtain sustained development and progress. Literature [12] believes that company performance is positively related to the use of innovative IT tools. If companies invest more in new technologies, this can increase the market share and market value of their products. Literature [13] believes that, in developed countries, investment in IT technology plays a very decisive role in production efficiency. Literature [14] believes that if a company has a comprehensive information system that can connect all departments within the company, this can reduce internal communication costs, improve information transmission efficiency, and improve business performance. Literature [15] points out that for the integration of business and finance to be realized in the enterprise, it must be supported by IT. Literature [16] puts forward the problem that the relevant information in the accounting information system cannot be shared in real time, and in order to solve 
the problem, the relevant financial process is redesigned. At the same time, it is pointed out that if the accounting goal is to be achieved under the new situation, the accounting business process must be reorganized. Literature [17] points out a set of innovative accounting information processing procedures based on the Internet by comparing the financial process under the value process and the financial process under the law. Literature [18] designs an accounting information system that can monitor financial information and business information in real time, with events and decisions as driving factors. Literature [19] believes that converting business information into financial information is the most important step in process reengineering. There are many conversion methods, for example, through business accounts, financially reviewed documents or those automatically generated by the accounting information system, and finally original vouchers provided by the accounting information system. Literature [20] puts forward the key points that should be paid attention to when the accounting information system is integrated with business and finance: one is to pay attention to the content, scope, and objectives involved in the establishment of business processes; the other is to formulate unified and standardized business processing rules.

\section{Data Mining Algorithm of Computerized Accounting}

Neurons can connect to each other and themselves, in addition to the connection between input neurons and reservoir neurons and that between output neurons. In addition, output neurons can also be self-connected and feedback-connected. We assume that the network has $\mathrm{K}$ inputs and represent the entire $\mathrm{K}$ inputs as a vector $u(n)=\left[u_{1}(n), u_{2}(n), \ldots, u_{k}(n)\right]$. If we assume that there are $\mathrm{N}$ neurons in the reserve pool, the corresponding state of the reserve pool neuron is $u_{i}(n)$. If we assume that the output dimension is $L$, the output is represented as a vector $\hat{y}(n)=\left[\hat{y}_{1}(n), \hat{y}_{2}(n), \ldots, \hat{y}_{L}(n)\right]$ as a whole. The connection weight matrix of the echo state network consists of four parts: (1) input connection matrix $W^{\text {in }}=\left(w_{i j}^{\text {in }}\right)$ with dimension $N \times K$, (2) internal connection matrix $W=\left(w_{i j}\right)$ with dimension $N \times N$ of the reserve pool, (3) output connection matrix $W^{\text {out }}=\left(w_{i j}^{\text {out }}\right)$ with dimension $L \times(K+N+\mathrm{L})$, and (4) output feedback matrix $W^{\text {back }}=$ $\left(w_{i j}^{\text {back }}\right)$ with dimension $N \times L$. For the echo state network used for iterative single-value prediction, the value of $L$ is 1 . The network topology of the echo state network is generated by the weight changes of neurons in the reserve pool. The status update expression and output expression of the network are as follows [21]:

$$
\begin{aligned}
& x(n)=f\left(W^{\text {in }} u(n)+W x(n-1)+W^{\text {back }} \hat{y}(n-1)\right), \\
& \hat{y}(n)=f^{\text {out }}\left(\left[u(n), x(n), \hat{y}(n-1) W^{\text {out }}\right]\right) .
\end{aligned}
$$

In (1), $f(*)=\left[f_{1}(*), f_{2}(*), \ldots, f_{N}(*)\right]$ is the excitation function of the internal module (usually the sigmoid function). The elements of the internal connection matrix $w$ must be randomly selected within $[-1,1]$, and the sparsity must be between $1 \%$ and $10 \%$. Moreover, in order to make the model stable and convergent, the spectral radius of $W$ should be less than 1 . In (2), $[u(n), x(n), \hat{y}(n-1)]$ is a composite vector composed of the input, the state corresponding to the neurons in the internal reserve pool, and the last network output. $f^{\text {out }}(*)=\left[f_{1}^{\text {out }}(*), f_{2}^{\text {out }}(*)\right.$, $\left.\ldots, f_{L}^{\text {out }}(*)\right]$ is the output function, and the identity function can be used. This can make the relationship between the matrix and the output a linear relationship, which can be solved more conveniently using parameter optimization methods, as shown in Figure 1.

Considering the special case of time series prediction, the output $y(n-1)$ at the previous moment can be merged into the input $u(n)$ at this moment. It can be seen that the output information at the last moment has been fed back at this moment. Therefore, the output feedback is no longer considered in the structure, and the output function takes the identity function. The pattern (1) and equation (2) can be simplified as follows:

$$
\begin{aligned}
& x(n)=f\left(w^{\text {in }} u(n)+W x(n-1)\right), \\
& \hat{y}(n)=[u(n), x(n)] W^{\text {out }} .
\end{aligned}
$$

Consider the case where the output length is 1 ; at this time, the dimension of $W^{\text {out }}$ is $(K+N) \mathrm{xl}$, and the form is changed from a matrix to a vector. It can be seen from the simplified formula that the nonlinear mapping between input and output is mainly completed by the neuron nonlinear activation function $f(*)=\left[f_{1}(*), f_{2}(*), \ldots\right.$, $f_{N}(*)$ ]. There is a linear relationship between the state vector $x(n)$ and the output $y(n)$. Writing in this way can simplify the derivation of subsequent formulas, making it easier for the identity output function to solve the model using parameter optimization methods, and the entire model needs to be calculated with only the output connection vector $W^{\text {out }}$, which requires less calculation.

Bootstrap interval estimation method is often used to solve the problem of small structure subsample test evaluation and infer the distribution characteristics of a certain statistic of data. It is suitable for some problems such as parameter interval estimation that are difficult to derive by conventional methods. The basic idea is to perform resampling with replacement in the original sample set $D_{\text {original }}$, and the sample set size obtained by sampling remains unchanged. That is, it is assumed that there are $M$ samples in the original sample set, the sample set obtained by sampling still has $M$ samples, and the probability of each sample being drawn is the same each time, which is $1 / M$. The resulting sample set is called the Bootstrap sample set. This extraction is repeated many times to obtain $B$ Bootstrap sample sets, which can be used as the training set of multiple models. In the prediction, multiple sets of model outputs can be obtained for the same input, and the required statistics to be estimated can be obtained.

Traditional Bootstrap interval estimation methods mainly include three types: standard Bootstrap, percentile Bootstrap, and $t$ percentile Bootstrap. 


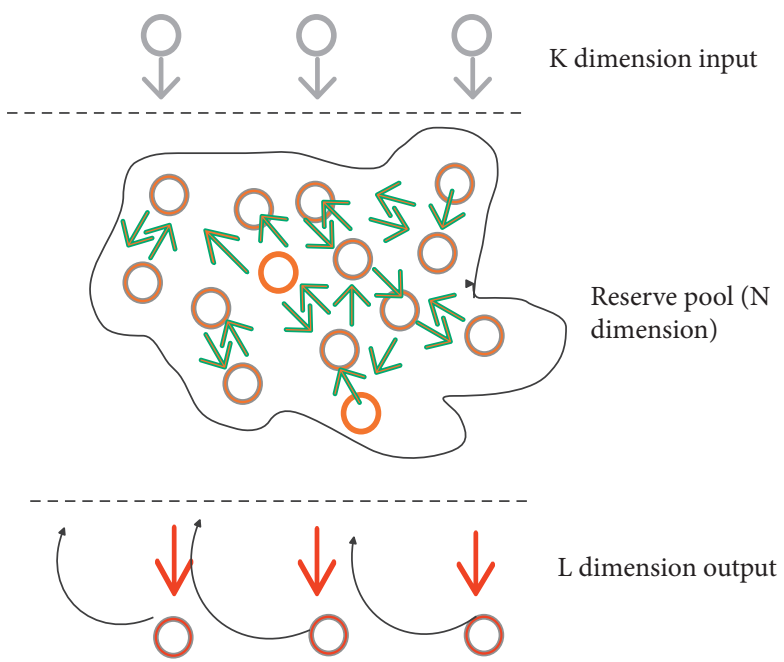

Figure 1: Echo status network.

3.1. Standard Bootstrap. Standard Bootstrap assumes that a sample with a capacity of $M$ is randomly selected independently from the original sample set $D_{\text {original }}$, and the sample is replaced each time. According to the idea of permutation and combination, there are at most $M M$ Bootstrap sample sets. By averaging the samples in each sample set, the value obtained is also an estimated value of the population mean, and the distribution of the sample mean is the same as the distribution of the population mean.

We assume that $\hat{y}$ is the estimated value of $y$ and that $\widehat{y}_{b}$ represents the mean value of the $b$-th Bootstrap sample set. Then, the mean and variance equations are

$$
\left\{\hat{y}=\frac{1}{B} \sum_{b=1}^{B} \hat{y}_{b}, \sigma_{\hat{y}}{ }^{2}=\frac{1}{B-1} \sum_{b=1}^{B}\left(\hat{y}_{b}-\widehat{y}\right)^{2} .\right.
$$

As a result, the confidence interval of the overall mean at the confidence level of $(1-\alpha) \%$ is

$$
\left[\widehat{y}-u_{1-\alpha / 2} \sigma_{\widehat{y}}^{2}, \hat{y}+u_{1-\alpha / 2} \sigma_{\hat{y}}^{2}\right] \text {. }
$$

Among them, $u_{1-\alpha / 2}$ is the $1-\alpha / 2$ percentile of the standard normal distribution.

3.2. Percentile Bootstrap. This method also requires Bootstrap sampling to obtain B Bootstrap sample sets and arrange the sample mean values obtained from each sample set in ascending order. It can get a set of Bootstrap sample means $\hat{y}_{b}(b=1,2,3, \ldots, B)$ with order. Then, the $\alpha / 2$-th and $1-\alpha / 2$-th points are the upper and lower limits of the confidence interval of the statistics under the $1-\alpha \%$ confidence level; namely,

$$
\left[\hat{y}\left(\frac{\alpha}{2} B\right), \hat{y}\left(1-\frac{\alpha}{2}\right) B\right] .
$$

3.3. T Percentile Bootstrap. This method is an improved method for percentiles. Generally, this method can get a more accurate confidence interval than the percentile Bootstrap method. It assumes that $B$ Bootstrap sample sets are drawn, and the $t$ statistic is calculated for the mean value of each sample set, as shown in the following formula:

$$
t_{b}^{*}=\frac{\widehat{y}_{b}-\hat{y}}{\sqrt{\operatorname{Var}(y)}} \text {. }
$$

It also assumes that $\hat{y}$ is the estimated value of $y$ and that $\hat{y}_{b}$ represents the mean value of the $b$-th Bootstrap sample set. It arranges the calculated mean values of each Bootstrap sample set in order to obtain the ordered Bootstrap sample set mean $\hat{y}_{b}(b=1,2,3, \ldots, B)$. The corresponding t-statistic sequence is $t_{b}^{*}(b=1,2,3, \ldots, B)$. When the significance level is $\alpha$, the $\alpha / 2$-th and $1-\alpha / 2$-th points are $\left[t^{*}((\alpha / 2) B), t^{*}(1-(\alpha / 2)) B\right]$, respectively, and the corresponding confidence interval is

$$
\left[\widehat{y}-t^{*}\left(\frac{\alpha}{2} B\right) \sqrt{\operatorname{Var}(y)}, \widehat{y}+t^{*}\left(1-\frac{\alpha}{2}\right) B \sqrt{\operatorname{Var}(y)}\right] .
$$

The method proposed in this paper uses the Tpercentile Bootstrap to refer to the predecessor's processing of the Bootstrap-based neural network interval prediction method in constructing the interval type.

The echo state network structure is simple, and most parameters only need to be randomly initialized. However, this also causes the shortcomings of the model's large randomness, the prediction effect is not stable enough, and most networks do not provide prediction intervals when making time series predictions. In view of the above shortcomings, some scholars integrate the network and make the model construct the prediction interval, which is the ESN integration model based on Bootstrap. This method finally achieved a better prediction effect. In summary, there are two bases for building an ESN integration model based on Bootstrap. One is the method idea of the bagging method. This method is a model integration method. The method is applied to the ESN integration model based on Bootstrap as explained below. The bagging method can effectively improve the stability and accuracy of the model, being 
especially suitable for input sensitive and random models, such as neural networks. We introduce the ESN integration model based on Bootstrap.

It can be seen from Figure 2 that the core of the model is an integrated body composed of B ESNs. We select $M$ samples and first perform Bootstrap sampling on the original sample set $D_{\text {original }}=\{u(n), t(n)\}_{i=1}^{M}$ composed of noise data during the training process. We get a sample set $D=\{D, D, \ldots, D\}$ consisting of B Bootstrap sample sets, with a total of $B \times M$ samples. Then, each sample subset corresponds to training an echo state network unit, $D_{b}=\left\{u_{b}(n), t_{b}(n)\right\}_{i=1}^{M}$ is the training set of the $b$-th echo state network; $u_{b}(n)$ is the input of the $b$-th network; $t_{b}(n)$ is the expected output value corresponding to the input of the $b$-th network; $y_{b}(n)$ is a single network output value driven by $u_{b}(n)$, which is also an estimate of $y(n)$; and $y(n)$ is the output value of the network integration model. The relevant expressions are summarized as follows:

$$
\begin{aligned}
x_{b}(n) & =f^{b}\left(w_{b}^{\text {in }} u_{b}(n)+W_{b} x_{b}(n-1)\right), \\
\hat{y}_{b}(n) & =\left[u_{b}(n), x_{b}(n)\right] w_{b}^{\text {out }}, \\
\hat{y}(n) & =\frac{1}{B} \sum_{i=1}^{B} \widehat{y}_{b}(n), \\
\sigma_{\widehat{y}}^{2}(n) & =E\left[(y(n)-\hat{y}(n))^{2}\right] \approx \frac{1}{B-1}(y(n)-\widehat{y}(n))^{2} .
\end{aligned}
$$

Equations (9) and (10) are the input and output expressions of a single echo state network. Among them, $x_{b}(n)$ is the state vector corresponding to the $b$-th echo state network at any time, $w_{b}^{\text {in }}$ is the input connection vector of the $b$-th echo state network, $W_{b}$ is the internal connection matrix of the $b$-th echo state network, and $w_{b}^{\text {out }}$ is the internal connection matrix of the $b$-th echo state network. The output connection matrix corresponding to the $b$ echo state networks, $f^{b}(*)=\left[f_{1}^{b}(*), f_{2}^{b}(*), \ldots, f_{N}^{b}(*)\right]$, is the reserve pool neuron activation function, all take the sigmoid function. Equation (11) describes the relationship between the true value estimate and the output value of each echo state network. This is also the key to the application of the bagging method; that is, the overall output of the model is the average output of multiple networks. Equation (12) is the expression of model randomness variance $\sigma_{\hat{y}}^{2}(n)$. If it is assumed that the echo state network model is the correct model behind the data, the randomness between the estimated value and the true value can be approximated to the randomness between the estimated value and the output of each echo state network.

Although the existing Bootstrap-based ESN ensemble model also has a good performance in the prediction effect, the method of maximizing the marginal likelihood is used in parameter estimation; instead of obtaining the parameters from the posterior distribution of the parameters, the performance of the estimated parameters is limited. Through theoretical study, it is found that there is a parameter estimation method that is more suitable for the model, that is, the parameter estimation method of variational reasoning.

When we set a training sample set, the maximum likelihood estimation can give the optimal parameters under a fixed likelihood distribution. However, when the sample size is small or there are outliers in the sample set, overfitting is easy to occur. The proposal of Bayesian estimation avoids the overfitting problem in principle. Bayesian estimation not only considers a single likelihood distribution, but also considers the prior distribution of the parameters in the model. The following specific expressions illustrate the differences between the two. If $Z$ is the model parameter set and $D$ is the training sample set, the following Bayesian formula can be listed:

$$
P(Z \mid D)=\frac{P(D \mid Z) P(Z)}{P(D)}
$$

Solving the maximum likelihood estimation is essentially solving the following optimal solution problem:

$$
\underset{z}{\arg \max } p(D \mid Z) \text {. }
$$

It can be seen that the above formula does not consider the prior distribution of the parameters, while the Bayesian estimation considers the elements of the entire posterior distribution. The expression for the maximum posterior probability is

$$
\underset{z}{\arg \max } \frac{P(D \mid Z) P(Z)}{P(D)} .
$$

Since $P(D)$ is a constant, it is not necessary to consider $P$ $(D)$ in the actual calculation, and only the product of the likelihood probability and the prior distribution of the parameter is used to solve the optimal value.

We assume that there is a Bayesian model in which each parameter has a prior probability distribution. These parameters can also have their own distribution parameters. All parameters in the model are recorded as the set $Z$. Similarly, denote the set of all observed samples as $D$. We assume that there are $n$ unknown parameters and $m$ observation samples; parameters are independent of each other; and samples are independent of each other. When the parameter set and sample set are represented as $Z=\left\{z_{1}, z_{2}, \ldots, z_{n}\right\}$ and $D=\left\{d_{1}, d_{2}, \ldots, d_{m}\right\}$, respectively, the probability distribution of the model can be further determined as $P(D, Z)$. The goal is to find an approximation to the posterior probability distribution $P(Z \mid D)$ and the evidence function $P(D)$. According to Bayesian formula and decomposing $P(D)$, there is the following formula:

$$
\ln P(D)=\ln \frac{P(D, Z)}{Q^{*}(Z)}-\ln \frac{P(Z \mid D)}{Q^{*}(Z)} .
$$

Among them, $Q^{*}(Z)$ is the joint probability distribution of the parameters to be estimated. When both sides of the equation are averaged for $Q^{*}(Z)$, we can get 


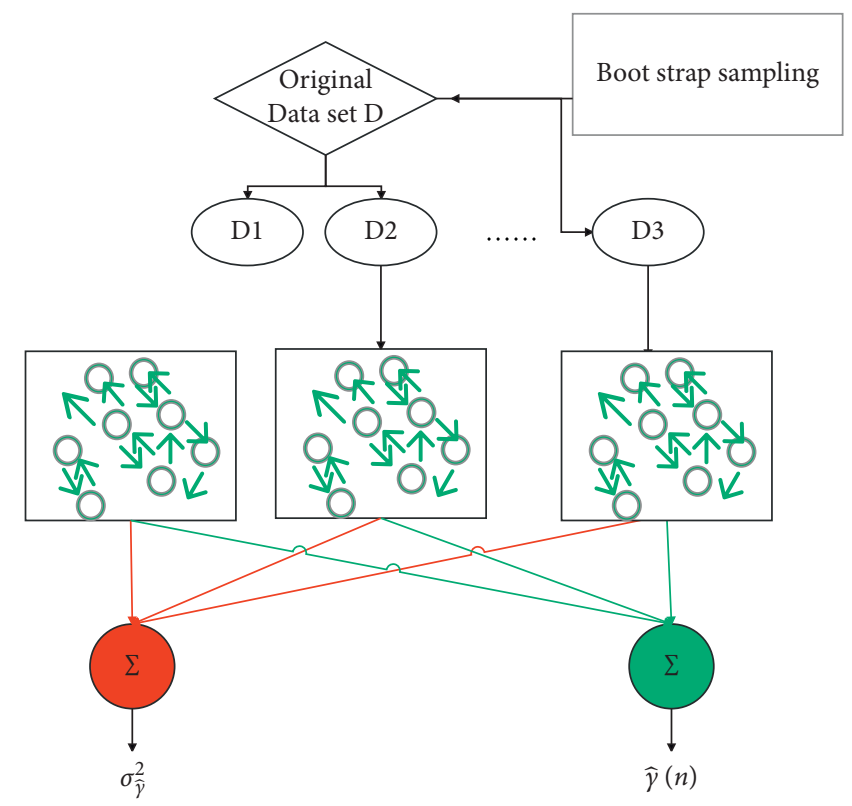

Figure 2: ESN integration model based on Bootstrap.

$$
\int Q^{*}(Z) \ln P(D) \mathrm{d} Z=\int Q^{*}(Z) \ln \frac{P(D, Z)}{Q^{*}(Z)} \mathrm{d} Z-\int Q^{*}(Z) \ln \frac{P(Z \mid D)}{Q^{*}(Z)} \mathrm{d} Z=\ln P(D) .
$$

The terms in the formula can be replaced by the following formula:

$$
\left\{\begin{array}{l}
L(Q)=\int Q^{*}(Z) \ln \frac{P(D, Z)}{Q^{*}(Z)} \mathrm{d} Z, \\
K L(Q \| P)=-\int Q^{*}(Z) \ln \frac{P(Z \mid D)}{Q^{*}(Z)} \mathrm{d} Z .
\end{array}\right.
$$

Then, the following expression is obtained:

$$
\ln P(D)=L(Q)+K L(Q \| P),
$$

In the formula, $L(Q)$ is called the lower variational bound, and $K L(Q \| P)$ is called the KL divergence, which represents the degree of similarity between the distribution $Q$ and the distribution $P$, with the value being greater than zero. When $L(Q)$ is larger, $K L(Q \| P)$ is smaller, and when $K L(Q \| P)$ reaches its minimum value, there is $Q^{*}(Z)=P(Z \mid D)$. At this time, the posterior distribution of the parameters can be approximated by $K L(Q \| P)$. According to the mean field theory, the parameter distribution is divided into the following forms:

$$
Q^{*}(Z)=\prod_{i=1}^{n} Q_{i}^{*}\left(Z_{i}\right)
$$

$Q_{i}^{*}\left(Z_{i}\right)$ is a certain type of distribution, such as normal distribution and gamma distribution, and the parameter distributions are independent of each other. The average field theory is derived from physics. It is a method of collectively processing the effects of objects in the environment and replacing individual effects with average effects. Through this kind of thinking, complex problems can be simplified. Below, by substituting (20) into $L(Q)$, the following derivation process can be obtained:

$$
\begin{aligned}
L(Q) & =\int \prod_{i} Q_{i}^{*}\left(Z_{i}\right)\left\{\ln P(D, Z)-\sum_{i} \ln Q_{i}^{*}\left(Z_{i}\right)\right\} \mathrm{d} Z \\
& =\int Q_{i}^{*}\left(Z_{i}\right)\left\{\ln P(D, Z)-\prod_{i \neq j} Q_{i}^{*}\left(Z_{i}\right) \mathrm{d} Z_{i}\right\} \mathrm{d} Z_{i}-\int Q_{i}^{*}\left(Z_{i}\right) \ln Q_{j}^{*}\left(Z_{j}\right) \mathrm{d} Z_{j} \\
& =\int Q_{i}^{*}\left(Z_{i}\right) \ln \widetilde{P}(D, Z) \mathrm{d} Z_{j}-\int Q_{j}^{*}\left(Z_{j}\right) \ln Q_{i}^{*}\left(Z_{i}\right) \mathrm{d} Z_{j}+\text { const. }
\end{aligned}
$$




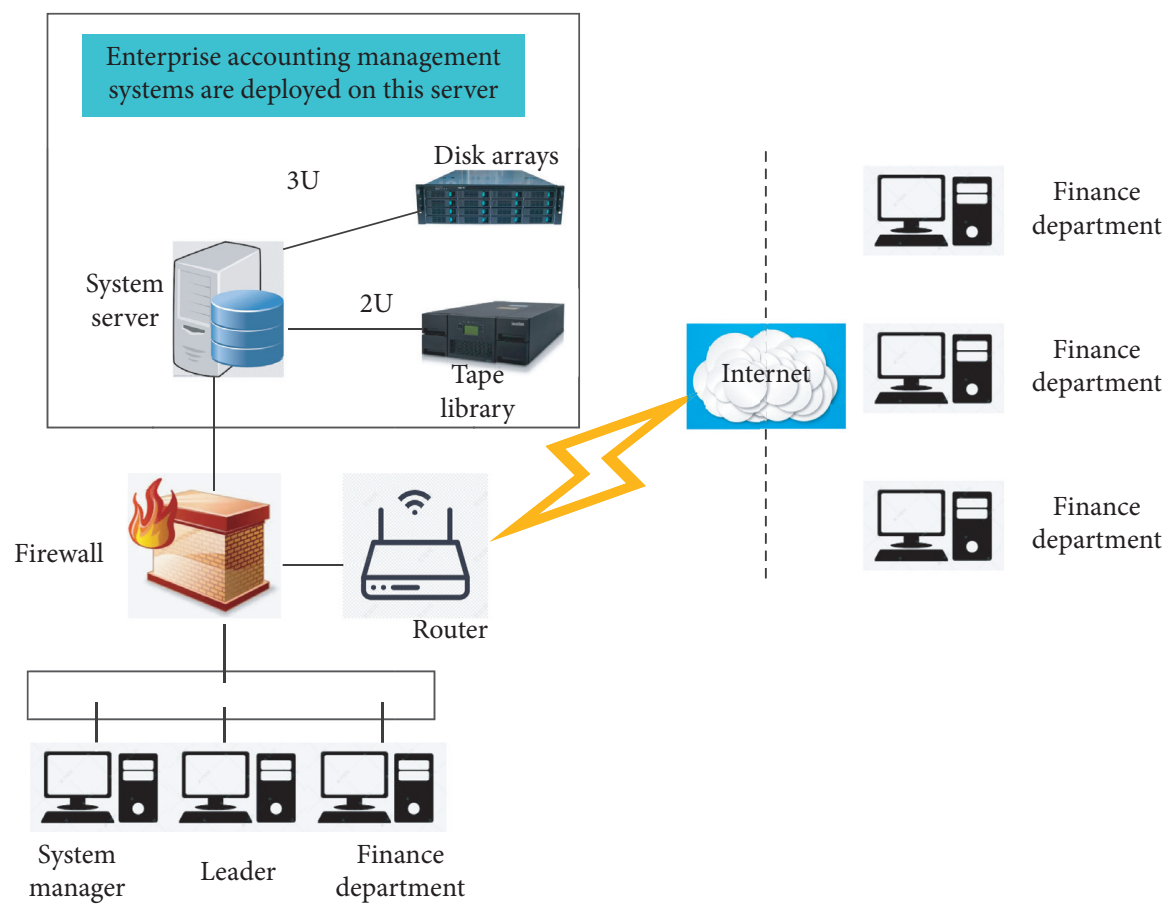

Network topology diagram of enterprise accounting management present system

FIGURE 3: Computerized intelligent accounting system based on big data technology.

Among them, const is a constant term, and $\widetilde{P}(D, Z)$ is a newly defined distribution, which represents the result distribution of integrating other parameters except $Z$ in the joint probability distribution $\widetilde{P}(D, Z)$. The expression is written as follows:

$$
\ln \widetilde{P}(D, Z)=E_{i \neq j}[\ln P(D, Z)]+\text { const. }
$$

Among them, $E_{i \neq j}[\ldots]$ means that $P(D, Z)$ integrates the parameters of all $Z(i \neq j)$, which is the expectation, which is specifically expressed as

$$
E_{i \neq j}[\ln P(D, Z)]=\int \ln P(D, Z) \prod_{i \neq j} Q_{i}^{*}\left(Z_{i}\right) \mathrm{d} Z_{i} .
$$

It can be seen in (21) that $L(Q)$ is the negative value of the KL divergence between $Q_{j}^{*}\left(Z_{j}\right)$ and $P\left(D, Z_{j}\right)$. Therefore, maximizing $L(Q)$ is equivalent to minimizing $\mathrm{KL}$ divergence. The smaller the KL divergence, the closer the two distributions are. Note that there is only parameter $\mathrm{Zj}$ in the distribution on the left side of (22). The general expression of $Q_{j}^{*}\left(Z_{j}\right)$ can be obtained as follows:

$$
\ln Q_{j}^{*}\left(Z_{j}\right)=E_{i \neq j}[\ln P(D, Z)]+\text { const. }
$$

In the formula, $E_{i \neq j}[\ln P(D, Z)]$ can be derived from the joint probability distribution of each parameter, then the distribution form of $Q_{j}^{*}\left(Z_{j}\right)$ is determined according to the form of the derivation result, and then the corresponding iterative formula is obtained through coefficient equivalence.

\section{Computerized Intelligent Accounting System Based on Big Data Technology}

The design goal of the enterprise accounting budget management system is that the system can efficiently complete the enterprise cost budget accounting work after the design is completed and ensure the normal and stable progress of the enterprise cost budget accounting work. This article combines big data technology to build a computerized intelligent accounting system based on big data technology, as shown in Figure 3.

The system structure diagram is shown as in Figure 4.

It can be seen from Figure 4 that the enterprise accounting management system is mainly divided into three levels, namely, the view layer, the logic layer, and the database layer.

The mechanism diagram of the system is shown in Figure 5.

In the enterprise accounting management system, because there are so many attributes of all entities, only a part of it is shown in the E-R diagram. According to the business process, the E- $\mathrm{R}$ diagram between each entity can be obtained, as shown in Figure 6.

Real-time accounting information system analysis is embodied in the processing of data. For real-time information system construction, it is the study of real-time database construction. After the data is stored in the database, the database is further divided into a model library, a knowledge base, and a method library. At the 


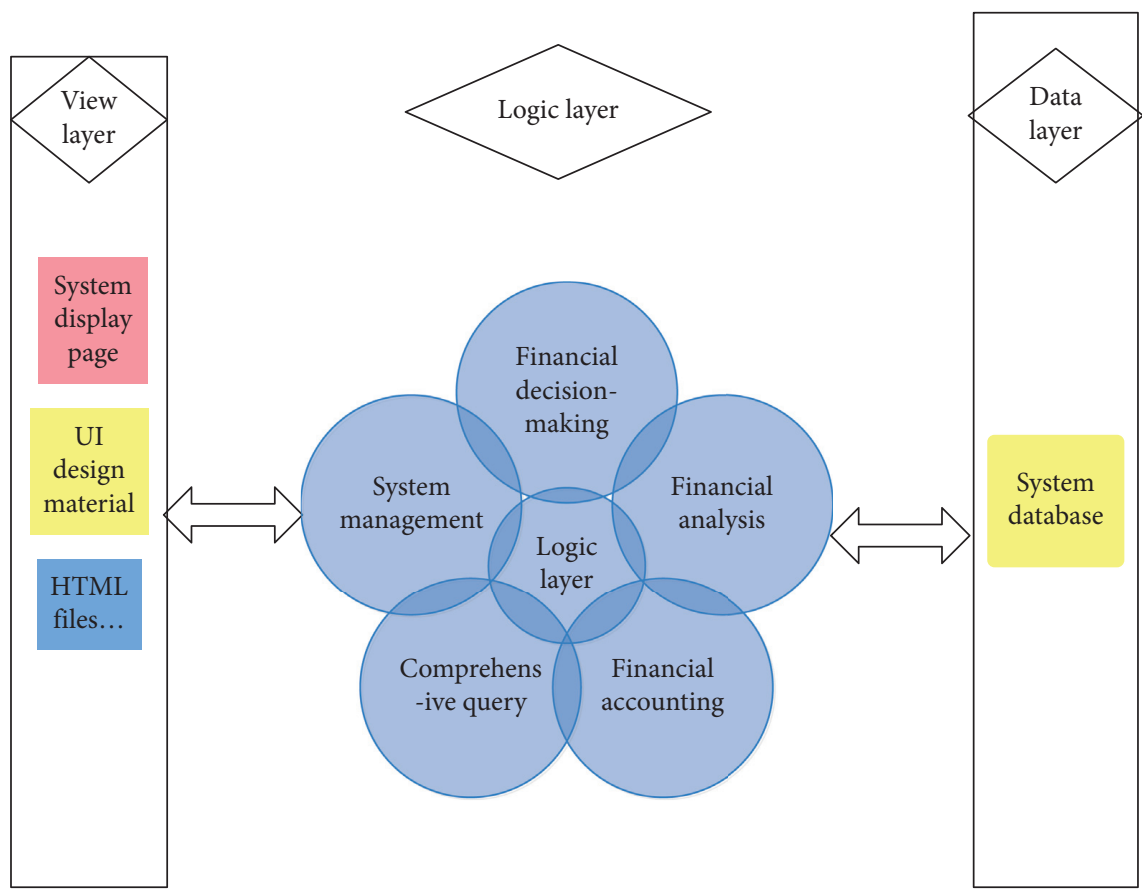

FIgURE 4: System architecture diagram.

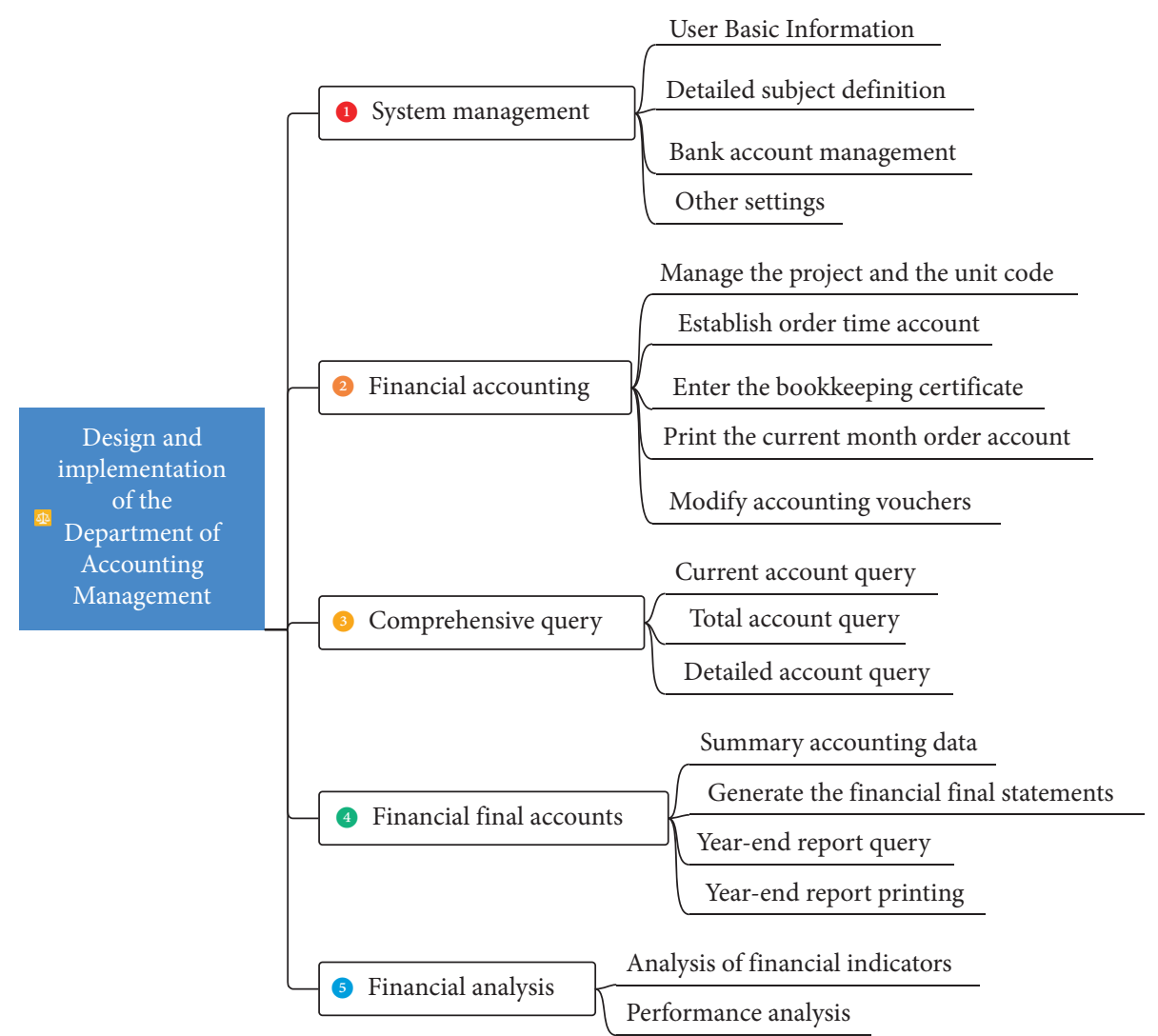

FIGURE 5: System function structure diagram.

same time, a series of data processing rules and standardized procedures are set for data extraction, transformation, and loading processing, which are loaded into the metadata database and data warehouse. Furthermore, when the system output interface has relevant command requirements, certain data analysis is performed to obtain the required view. The real-time database model is shown in Figure 7. 


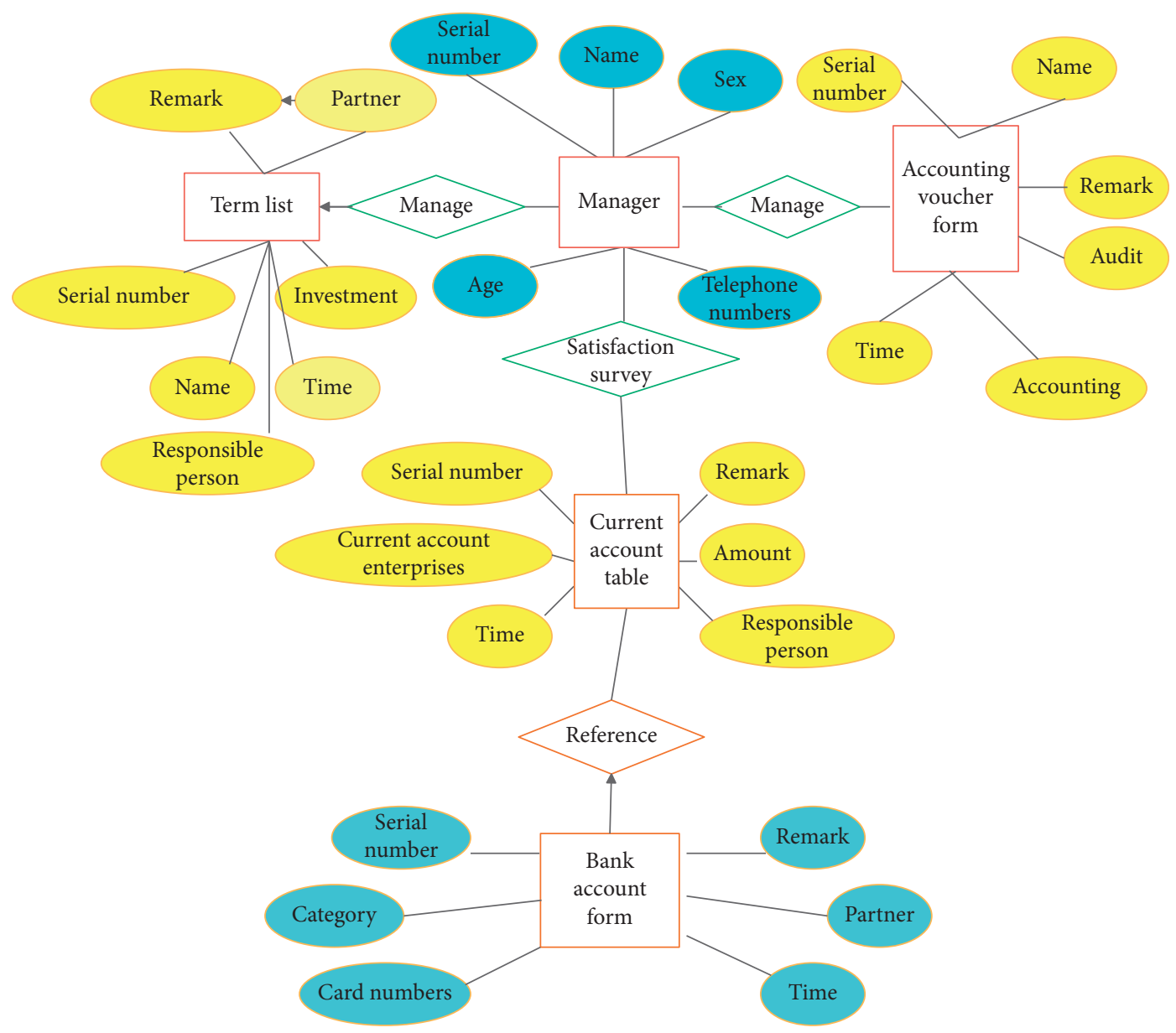

FIGURE 6: E-R diagram of the accounting management system.

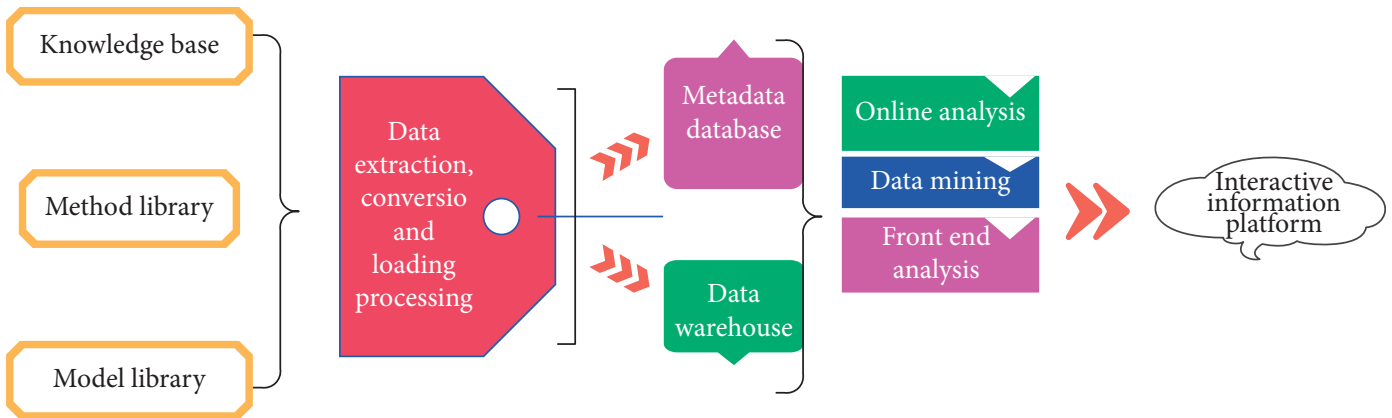

Figure 7: Real-time database model diagram.

In order to rationally allocate corporate resources, optimize business processes, and reduce human resource costs, its profound connotation lies in the use of network and computer technology to centralize management, optimize configuration, and monitor feedback to realize data sharing and gradually realize the reform of enterprise information system processes. After the above logical design is completed, it is necessary to design the physical storage process of the data, that is, the actual application of the above process, which will not be described here. The final construction system is shown in Figure 8.
After constructing the above accounting information system, this paper conducts performance verification of the system. Moreover, this article combines the accounting requirements under the background of big data to analyze the system performance. In addition, this paper conducts data mining through multiple sets of data from a certain company, conducts intelligent accounting to calculate the effects of data mining and intelligent accounting, and evaluates them, and the test results shown in Table 1 and Figure 9 are obtained.

Through the above experimental research, it can be verified that the computerized intelligent accounting system 


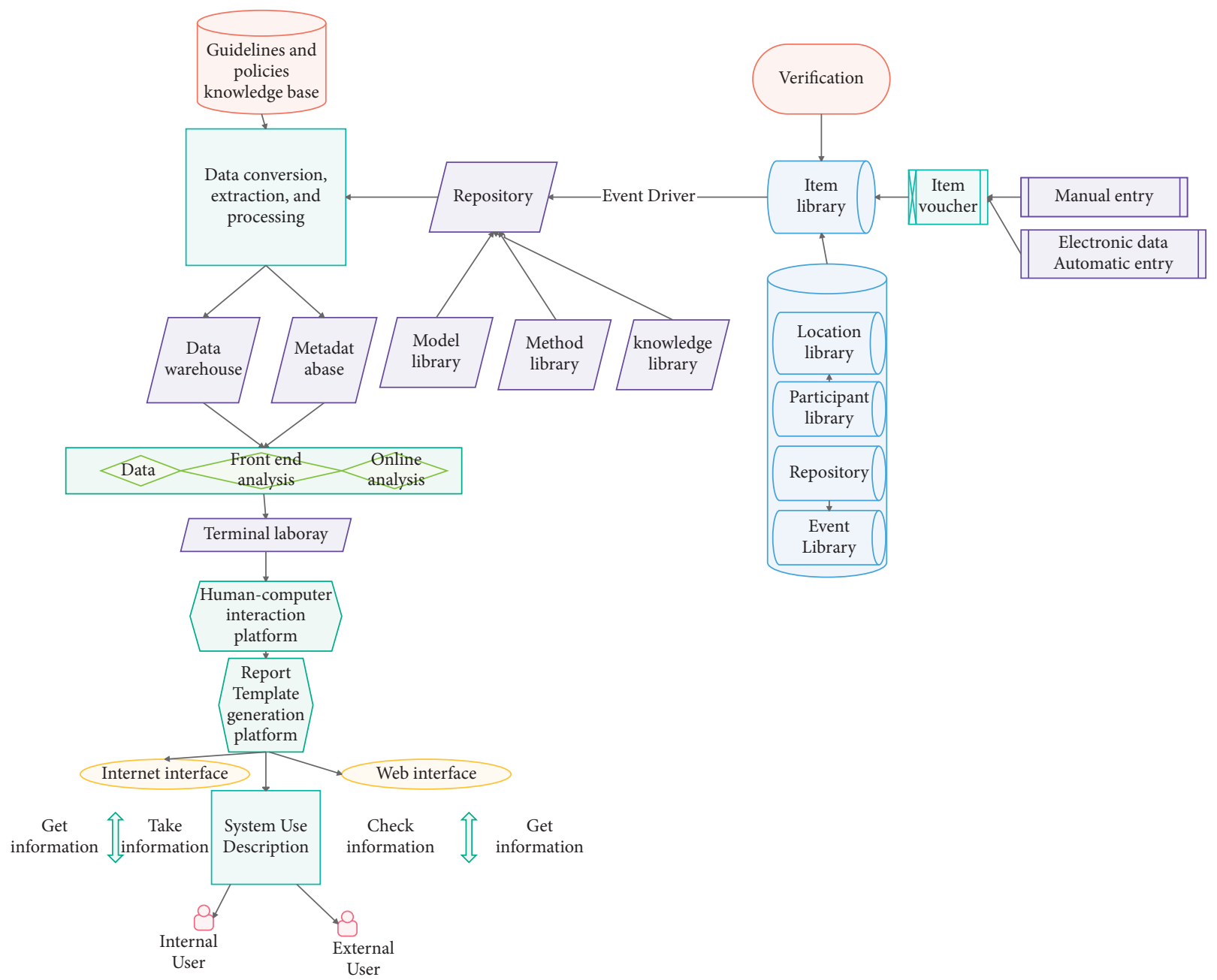

FIgURE 8: Structure diagram of accounting information system.

TABLe 1: Performance statistics of the computerized intelligent accounting system based on big data analysis.

\begin{tabular}{|c|c|c|c|c|c|c|c|c|}
\hline No. & Data mining & Smart accounting & No. & Data mining & Smart accounting & No. & Data mining & Smart accounting \\
\hline 1 & 89.02 & 89.29 & 23 & 96.34 & 85.74 & 44 & 93.76 & 79.48 \\
\hline 2 & 96.45 & 89.56 & 24 & 90.21 & 83.36 & 45 & 93.16 & 87.05 \\
\hline 3 & 96.78 & 79.02 & 25 & 94.55 & 82.22 & 46 & 91.12 & 89.31 \\
\hline 4 & 92.75 & 86.04 & 26 & 95.84 & 87.27 & 47 & 90.04 & 84.61 \\
\hline 5 & 94.32 & 89.74 & 27 & 93.98 & 90.79 & 48 & 89.47 & 84.07 \\
\hline 6 & 91.46 & 84.60 & 28 & 92.67 & 87.88 & 49 & 95.02 & 89.98 \\
\hline 7 & 96.70 & 88.04 & 29 & 94.32 & 85.93 & 50 & 96.03 & 84.13 \\
\hline 8 & 90.83 & 88.87 & 30 & 89.27 & 89.17 & 51 & 94.94 & 84.25 \\
\hline 9 & 94.66 & 85.64 & 31 & 92.57 & 81.79 & 52 & 91.92 & 79.25 \\
\hline 10 & 93.02 & 85.88 & 32 & 95.00 & 87.25 & 53 & 93.33 & 82.07 \\
\hline 11 & 94.65 & 86.72 & 33 & 94.85 & 84.83 & 54 & 91.17 & 85.12 \\
\hline 12 & 92.74 & 88.20 & 34 & 94.10 & 81.43 & 55 & 91.35 & 85.26 \\
\hline 13 & 96.23 & 84.23 & 35 & 91.85 & 88.30 & 56 & 89.81 & 83.56 \\
\hline 14 & 88.96 & 86.47 & 36 & 91.07 & 84.53 & 57 & 93.84 & 84.47 \\
\hline 15 & 89.99 & 80.85 & 37 & 96.45 & 86.46 & 58 & 93.47 & 88.16 \\
\hline 16 & 90.97 & 80.15 & 38 & 94.44 & 87.18 & 59 & 90.67 & 81.74 \\
\hline 17 & 93.36 & 85.85 & 39 & 88.60 & 80.73 & 60 & 88.15 & 86.73 \\
\hline 18 & 95.65 & 79.50 & 40 & 92.05 & 84.98 & 61 & 90.37 & 81.44 \\
\hline 19 & 89.25 & 87.48 & 41 & 93.07 & 83.76 & 62 & 88.87 & 85.27 \\
\hline 20 & 93.40 & 87.14 & 42 & 96.45 & 87.91 & 63 & 92.68 & 85.75 \\
\hline 21 & 91.13 & 87.37 & 43 & 89.98 & 81.67 & 64 & 93.88 & 90.69 \\
\hline 22 & 91.97 & 79.52 & & & & & & \\
\hline
\end{tabular}




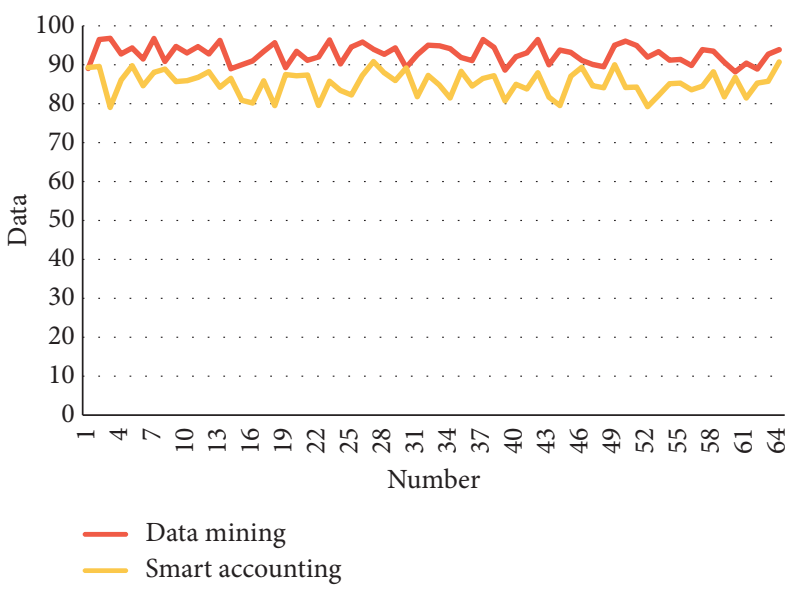

Figure 9: Statistical diagram of system experiment data.

based on big data technology constructed in this article has a certain effect.

\section{Conclusion}

Because the financial informatization system and business informatization system are generally constructed in batches and by departments and there is also a lack of unified strategic planning during the construction, most enterprises' financial informatization and business informatization are in a state of disconnected systemic management. At the same time, the issue of corporate "information islands" has also emerged. During the promotion of management accounting, due to the lack of support from a unified information system, the integration of business and finance has become an empty talk, and the manual management model has made it difficult for management accounting tools to exert their value. With the continuous development of enterprises, business and financial conflicts and contradictions have become increasingly prominent. Moreover, the business always ignores the company's financial system regulations for various reasons and goes beyond the company's system and financial discipline in daily business activities. Finance, of course, always carries the banner of internal risk control, uses excessive supervision powers to prevent audit and supervision risks, and dictates specific business activities, which makes it difficult to implement some special businesses and even causes companies to miss development opportunities. This article combines the big data technology to construct the accounting computerization system, obtains the scientific and effective accounting information processing results through intelligent big data processing, and improves the accounting management efficiency on this basis. The experimental research results show that the computerized intelligent accounting system based on big data technology constructed in this paper has a certain effect.

\section{Data Availability}

The labeled datasets used to support the findings of this study are available from the corresponding author upon request.

\section{Conflicts of Interest}

The authors declare no conflicts of interest.

\section{Acknowledgments}

This study was sponsored by Tangshan Normal University.

\section{References}

[1] P. Rajsic, A. Weersink, A. Navabi, and K. P. Pauls, "Economics of genomic selection: the role of prediction accuracy and relative genotyping costs," Euphytica, vol. 210, no. 2, pp. 1-18, 2016.

[2] F. Jahedpari, T. Rahwan, S. Hashemi et al., "Online prediction via continuous artificial prediction markets," IEEE Intelligent Systems, vol. 32, no. 1, pp. 61-68, 2017.

[3] V. Daksiya, H. T. Su, Y. H. Chang, and E. Y. M. Lo, "Incorporating socio-economic effects and uncertain rainfall in flood mitigation decision using MCDA," Natural Hazards, vol. 87, no. 1, pp. 515-531, 2017.

[4] S. Lahmiri, "A variational mode decompoisition approach for analysis and forecasting of economic and financial time series," Expert Systems with Applications, vol. 55, no. 8, pp. 268-273, 2016.

[5] N. Gordini, "A genetic algorithm approach for SMEs bankruptcy prediction: empirical evidence from Italy," Expert Systems with Applications, vol. 41, no. 14, pp. 6433-6445, 2014.

[6] A. Ferramosca, A. H. González, D. Limon, and D. Limon, "Offset-free multi-model economic model predictive control for changing economic criterion," Journal of Process Control, vol. 54, no. 3, pp. 1-13, 2017.

[7] C. J. A. Jane, "Hybrid model combined grey prediction and autoregressive integrated moving average model for talent 
prediction," Journal of Grey System, vol. 21, no. 2, pp. 91-102, 2018.

[8] N. A. Khadjeh, S. Aghabozorgi, W. T. Ying, and D. C. L. Ngo, "Text mining for market prediction: a systematic review," Expert Systems with Applications, vol. 41, no. 16, pp. 76537670, 2014.

[9] M. Ellis and P. D. Christofides, "Integrating dynamic economic optimization and model predictive control for optimal operation of nonlinear process systems," Control Engineering Practice, vol. 22, no. 1, pp. 242-251, 2014.

[10] W. Schultz, W. R. Stauffer, and A. Lak, "The phasic dopamine signal maturing: from reward via behavioural activation to formal economic utility," Current Opinion in Neurobiology, vol. 43, no. 5, pp. 139-148, 2017.

[11] W. Montford and R. E. Goldsmith, "How gender and financial self-efficacy influence investment risk taking," International Journal of Consumer Studies, vol. 40, no. 1, pp. 101-106, 2016.

[12] W. S. Muthii, J. G. Wanjohi, and J. M. Ndambiri, "The effect of financial risk management on the financial performance of commercial banks in Kenya," International Journal of Finance and Banking Research, vol. 3, no. 5, pp. 70-81, 2017.

[13] J. Magendans, J. M. Gutteling, and S. Zebel, "Psychological determinants of financial buffer saving: the influence of financial risk tolerance and regulatory focus," Journal of Risk Research, vol. 20, no. 8, pp. 1076-1093, 2017.

[14] E. S. Cole, D. Walker, A. Mora, and M. L. Diana, "Identifying hospitals that may be at most financial risk from medicaid disproportionate-share hospital payment cuts," Health Affairs, vol. 33, no. 11, pp. 2025-2033, 2014.

[15] A. Zalik, "Resource sterilization: reserve replacement, financial risk, and environmental review in Canada's tar sands," Environment and Planning: Economy and Space, vol. 47, no. 12, pp. 2446-2464, 2015.

[16] C. Lucarelli, P. Uberti, and G. Brighetti, "Misclassifications in financial risk tolerance," Journal of Risk Research, vol. 18, no. 4, pp. 467-482, 2015.

[17] E. Y. Chan, "Physically-attractive males increase men's financial risk-taking," Evolution and Human Behavior, vol. 36, no. 5, pp. 407-413, 2015.

[18] T. West and A. C. Worthington, "Macroeconomic conditions and Australian financial risk attitudes, 2001-2010," Journal of Family and Economic Issues, vol. 35, no. 2, pp. 263-277, 2014.

[19] T. K. Scharding, "Imprudence and immorality: a kantian approach to the ethics of financial risk," Business Ethics Quarterly, vol. 25, no. 2, pp. 243-265, 2015.

[20] J. Su and E. Furman, "A form of multivariate pareto distribution with applications to financial risk measurement," ASTIN Bulletin, vol. 47, no. 1, pp. 331-357, 2017.

[21] L. John, "Luck, Justice and systemic financial risk," Journal of Applied Philosophy, vol. 34, no. 3, pp. 331-352, 2017. 